\title{
HIV treatment, care and support in NSW: a work necessarily still in progress
}

\author{
Roger Garsia \\ Royal Prince Alfred Hospital, Sydney \\ Email: roger.garsia@email.cs.nsw.gov.au
}

\begin{abstract}
A diverse range of services were developed in NSW in response to the emerging HIV epidemic. These services included innovative multidisciplinary service models of care most of which have been durable. Allied health teams and ancillary services funded by non-government organisations have played a vital role in delivering care and support in the community. A consistency of approach to treatment across the health sector has been reinforced through continuing education activities and credentialing of antiretroviral-drug prescribers. This investment in care and treatment has resulted in reduced hospital bed use. High levels of treatment uptake over many years are likely to have a favourable impact on transmission rates thus contributing to the stability of the epidemic in NSW.
\end{abstract}

Over the last 25 years, human immunodeficiency virus (HIV) has challenged those planning and delivering health care in Australia. New South Wales (NSW) has been the most severely and disproportionately affected by HIV with the highest absolute numbers of HIV/AIDS diagnoses, deaths, hospital admissions and, until 2009, annual new HIV diagnoses per capita. ${ }^{1}$ Responding to this situation, from the earliest awareness of acquired immune deficiency syndrome (AIDS) in Sydney residents ${ }^{2,3}$ until the present, the health-care system in NSW has been innovative and has consistently delivered state of the art diagnostic, medical and nursing care. NSW has arguably led best practice in many domains. While the state's well documented record in prevention has been widely lauded, ${ }^{4}$ less well known is the approach to care and treatment which, combined with the success in primary HIV prevention, has resulted in extremely low rates of AIDS in recent years. ${ }^{1}$ This article describes how treatment and care, like prevention strategies, evolved in response to developments in treatment and the subsequent changes in patterns of illness.

\section{Development of dedicated HIV services in NSW}

In a public health-focused response to the AIDS epidemic in $1989,{ }^{5}$ Australia was the first country to publish a comprehensive HIV/AIDS strategy. The first strategy and its five revisions have been informed by a diverse group of stakeholders. NSW Government responses and operational plans for combating HIV were similarly developed through the collaborative efforts of multiple tiers of government, medical and scientific practitioners and nongovernment organisations (NGOs) with broad constituencies. These plans have embodied the principles enunciated in the national strategies in addressing the demography, behaviour characteristics and sensitivities displayed in the NSW population. ${ }^{6}$ Moving from ad hoc supplementary funding in the early 1980 s during the initial phase of the HIV epidemic, formal health-care planning processes and research funding rapidly ensued. A strong laboratory base in microbiology and immunology, available through the tax-funded universal health-care system, provided a sound foundation for HIV care across both public and private sectors.

The NSW Health-administered funding stream aimed to support centres of excellence in clinical care and research, and to ensure provision of high quality statewide, decentralised and accessible testing and treatment at sexual health centres and designated clinics linked to, or sited at, the principal referral hospitals. Looking back, the rollout of enhanced capacity in infectious diseases and microbiology, immunology, sexual health and in the supporting allied-health disciplines was achieved quite rapidly. Designated hospital and hospice beds were nominated and specialist positions in the above disciplines were expanded, adequately increasing the capacity to deal with the most urgent and severe problems in hospital. ${ }^{7}$ With the help of a small group of somewhat overburdened general practitioners (GPs), the shared-care model of care was able to operate, with GPs referring those needing hospital care and providing high-quality follow-up care in the community. Subsequently, much effort went into building a broader, community-based, primary-care capacity and enhancing the allied health and nursing support for those people with HIV experiencing physical and mental disability and suffering the effects of grief, poverty and social dislocation. 'Hospital-in-the-home' models of care were 
explored and nursing expertise in all aspects of HIV care was fostered. Specialist HIV clinics and services, including in some cases palliative care, have been durable and remain extremely multidisciplinary.

The suite of services which emerged across area health services differed, not only between area health services but also within them. ${ }^{7}$ These still reflect HIV service needs dictated by demography, geography and the availability of expertise. The planning documents on which the current configuration of services were initially based assumed that the increasing demand on services would continue and that decentralised HIV care in all tertiary hospitals would be necessary to cater for the projected expanding workload.

\section{Successful treatment regimens}

At the peak of the incidence of AIDS in NSW in the mid1990s and prior to the widespread uptake of combination antiretroviral therapy (cART), life expectancy after a diagnosis of AIDS was generally less than 2 years. The need to prepare for diminishing health and a shortened working life was evident. ${ }^{8}$ The success of cART regimens in containing viral load and enabling immunological reconstitution led to sustained survival. Consequently care and treatment needs changed dramatically from the mid 1990s. ${ }^{9}$ Evidence of this change could be seen in reduced bed use, rising demands on ambulatory care services as numbers on treatment rose, and fewer referrals to physicians experienced in end-of-life care. ${ }^{10,11}$ Ageing-related morbidity, complex medication regimens needed to deal with antiviral drug resistance and dysregulated metabolism from treatment side-effects increasingly became the dominant issues in HIV management of the long-time survivors of HIV. ${ }^{10}$

\section{Statewide services}

As the epidemic of HIV evolved and reached beyond those populations initially most affected, new services with a statewide role were encouraged to develop or expand to meet the specific needs of various groups. The Paediatric HIV Service at Sydney Children's Hospital, the TransfusionRelated HIV/ AIDS Service at Westmead Hospital, the NSW Haemophilia referral service at Royal Prince Alfred Hospital and the Multicultural HIV Education Service in Central Sydney are examples of the diversity of early statewide services that emerged.

The role of statewide services has been periodically reviewed. Following the most recent review, reconfigurations have been recommended and implemented for some services. Review, renewal and refocusing of resources, the latter partly through HIV program-specific resource distribution formula mechanisms, has been a strength of the NSW HIV service planning and governance process. Good use has been made of the interest and expertise of stakeholders brought together in the NSW Ministerial Advisory Committee on HIV and Sexually Transmissible Infections and its associated committee which advises on hepatitis.

\section{Practicalities of shared care}

If we reflect on the decades of care of HIV, the severity of illness faced by the first wave of AIDS patients necessitated tertiary level care in most cases, whereas people acquiring HIV more recently are likely to have most of their monitoring and management in primary-care settings, reflecting their encouraging long-term prognosis on treatment. $^{12}$ It is pertinent that soon after antiretroviral drugs were registered, NSW was proactive in credentialing primary-care and hospital-based specialists in the prescription of antiretroviral therapy. Those and other subsidised Section 100 drugs involved in HIV care were therefore accessible from public hospital pharmacies. Credentialing courses and assessments were initially undertaken inhouse and then by the Australasian Society for HIV Medicine for NSW. Experienced practitioners from hospital and primary-care practices helped to prepare manuals and lecture, tutor, supervise and mentor colleagues as they upgraded their skills in the management of HIV. These courses facilitated system-wide consistency in the delivery of HIV treatment and were delivered through a multidisciplinary approach to continuing education.

NSW clinicians were also fortunate in having abundant expertise in the diverse aspects of HIV management due to their proximity to the HIV geographical epicentre, their historical involvement in dealing with the onset of the HIV epidemic and their awareness of ongoing scientific advances in the basic and applied sciences at the National Centres of HIV research (clinical, epidemiological, social and virological).

Nonetheless there remains in NSW geographical patchiness in the availability of GPs who are expert in HIV management. ${ }^{7}$ The issues facing primary-care practitioners with low HIV caseloads, many operating out of large multimember practices, are mirrored in some low caseload tertiary centres. Here, due to the welcome impact of cART, specialist trainees see little of the complex management required to treat some of the less frequent complications of HIV. Recently, in an effort to better encourage continuity of patient care in the community and sustain contact with the diagnosing practitioner, a formal trial of mentoring began under the auspices of the NSW Department of Health and the Australasian Society for HIV Medicine. This initiative aims to link HIV inexperienced practitioners making a new HIV diagnosis with more experienced clinicians who are able to provide them with advice and information. 
In the hospital sector, a strategy of decentralised specialist HIV referral services may not be sustainable beyond the next decade. Fortunately at present there are sufficient numbers of highly-experienced personnel and a willing group of trainees aware of the issues. These trainees are working to broaden their experience both locally and internationally and are well placed to continue to deliver high-level expertise in treating HIV well into the future.

\section{Innovation in-service delivery}

\section{AIDS Dementia and HIV Psychiatry Service}

Novel collaborations based around the neurology unit at Sydney's St Vincent's Hospital and the psychiatry unit at Royal Prince Alfred Hospital developed a new model of care. It involved three arms:

- community support for those with mild HIV neurocognitive decline

- full time nursed residential support for those with more severe forms at The Bridge, a residential city-fringe dementia service for HIV

- expansion of hospice dementia services at the inner city Sacred Heart Hospice for those with advanced dementia.

The AIDS Dementia and HIV Psychiatry Service provides statewide outreach which has been an innovative and successful means of case managing and brokering support for many HIV patients living in their own or their carers' residences.

\section{Coordinated community health support}

NSW has also been innovative in promoting coordinated community health support for those with HIV in the community. Pilot services based on allied health workers providing nursing, counselling, physiotherapy, occupational therapy, dietary advice, drug and alcohol support and psychiatric care are still being expanded according to need. Volunteer organisations supporting the Ankali project, Bobby Goldsmith Foundation and various faith-based services are still frequently called on to provide practical assistance beyond that provided by health services. The larger NGOs, principally ACON (AIDS Council of NSW), Positive Life NSW and the NSW Users and AIDS Association, partly supported by NSW government grants, have been dynamic, important and vigorous participants in support service provision to their respective client bases.

\section{Planning for change}

With the benefit of hindsight, one can question whether the hybrid models which evolved in NSW were in all cases the most appropriate ones for the scale and nature of the epidemic. To explore that issue contemporaneously a series of care and treatment reviews has been undertaken independently, funded by NSW Department of Health, to gather and analyse views from health-care professionals and the community. No strong recommendations for radical change in the pattern of service development emerged from those reviews and, although gaps were identified in service provision, largely emanating from federal-state responsibility overlap issues (for example dental care), no major flaws with the current model of care have been identified. Following recent reviews, service performance monitoring has been enhanced and a better documentation and understanding of service delivery has flowed from consistent reporting of activity and more reliable recording of basic aggregated demographics of the clientele and activity of these services.

\section{Looking into the future}

The decline in bed use experienced through the 1990s probably reached its nadir early in the 2000s and there is a strong suggestion that the current overall HIV-related health service use has reached a stable level. The challenges ahead are numerous and involve adjusting to the changing pattern of HIV disease in NSW and internationally. ${ }^{12}$ Late presentation, which is not diminishing, makes for ongoing challenges in care and treatment. ${ }^{13}$ Each new pharmaceutical agent elicits a new range of patient management issues; recent release of additional antiretroviral agents acting on the HIV-driven chromosomal integration process and viral entry blockers may contribute to even greater impact on HIV disease ${ }^{14}$ and interruption of transmission than achieved through previous cART regimens. Thus service needs may further change if these agents achieve widespread use and prove to have both sustained potency and lower levels of metabolic and other unwanted side-effects than their predecessors.

\section{Conclusion}

The success of care and treatment programs in NSW over the last 25 years is now a matter of record. ${ }^{15}$ There are strong grounds for expecting the pattern of HIV treatment and care to be stable into the next decade. In NSW the experience of most treated patients in 2010 is one of health maintenance and chronic disease management with an expectation of relatively low levels of medium-term morbidity and a lifespan approaching normal. ${ }^{12}$ For that outcome, following 25 years of intervention at a population level, everyone involved over the years can be both grateful and proud. For those now at the coalface there are many issues to confront such as the current needs of older HIV-positive patients, the management of HIV-associated malignancies, the dilemmas in treatment initiation timing for those who have more recently acquired the infection, treatment of co-infection for those people with HIV and hepatitis 
viruses and catering for the support needs of HIV patients with disadvantaged social circumstances.

The times ahead will undoubtedly provide new and ongoing challenges well into the current decade and beyond. Hopefully success in the past will provide the impetus and the confidence necessary for successful innovation in the future.

\section{References}

1. National Centre in HIV Epidemiology and Clinical Research. HIV/AIDS, viral hepatitis and sexually transmissible infections in Australia Annual Surveillance Report 2009. National Centre in HIV Epidemiology and Clinical Research, The University of New South Wales, Sydney, NSW, 2009. Available at: http:// www.nchecr.unsw.edu.au/NCHECRweb.nsf/page/Annual+ Surveillance+Reports (Cited 10 March 2010).

2. Mutton K, Gust I. Acquired immune deficiency syndrome. Med J Aust 1983; 1(12): 540-1.

3. Dalgleish AG, Prentice RL, Gatenby PA, Loblay RH, Callard RE, Basten A. Acquired immune deficiency syndrome. A prodromal form. Med J Aust 1983; 1(12): 558-60.

4. Gupta GR, Parkhurst JO, Ogden JA, Aggleton P, Mahal A. Structural approaches to HIV prevention. Lancet 2008; 372: 764-75. doi:10.1016/S0140-6736(08)60887-9

5. Commonwealth of Australia. AIDS: a time to care, a time to act: towards a strategy for Australians. Canberra: Australian Government Publishing Service; 1988.

6. Imrie J, Frankland A, editors. HIV/AIDS, hepatitis and sexually transmissible infections in Australia: annual report of trends in behaviour 2008. Sydney: National Centre in HIV Social Research, University of New South Wales; 2008.

7. Hardwick J, Cotton R. HIV AIDS care and treatment service needs assessment. Sydney: NSW Department of Health; 2005. Available at: http://www.health.nsw.gov.au/pubs/2005/pdf/ hivaids_care_assess_1-66.pdf (Cited 10 March 2010.)

8. Collaborative Group on AIDS Incubation and HIV Survival including the CASCADE EU Concerted Action. Concerted Action on SeroConversion to AIDS and Death in Europe. Time from HIV-1 seroconversion to AIDS and death before widespread use of highly active antiretroviral therapy: a collaborative reanalysis. Lancet 2000; 355: 1131-7. doi:10.1016/S0140-6736 (00)02061-4

9. Antiretroviral therapy cohort collaboration (ART-CC). Prognosis of patients treated with cART from 36 months after initiation, according to current and previous CD4 count and plasma HIV-1 RNA measurements. AIDS 2009; 23: 2203-12.

10. Cooper DA. Life and death in the cART era. Lancet 2008; 372 : 266-7. doi:10.1016/S0140-6736(08)61086-7

11. Antiretroviral therapy cohort collaboration (ART-CC). HIV treatment response and prognosis in Europe and North America in the first decade of highly active antiretroviral therapy: a collaborative analysis. Lancet 2006; 368: 451-8. doi:10.1016/ S0140-6736(06)69152-6

12. Antiretroviral therapy cohort collaboration (ART-CC). Life expectancy of individuals on combination antiretroviral therapy in high income countries: a collaborative analysis of 14 cohort studies. Lancet 2008; 372: 293-9. doi:10.1016/S0140-6736(08) 61113-7

13. Guy RJ, McDonald AM, Bartlett MJ, Murray JC, Giele CM, Davey TM et al. Characteristics of HIV diagnoses in Australia, 1993-2006. Sex Health 2008; 5: 91-6. doi:10.1071/SH07070

14. Lennox JL, DeJesus E, Lazzarin A, Pollard RB, Madruga JV, Berger DS et al. Safety and efficacy of raltegravir-based versus efavirenz-based combination therapy in treatment-naive patients with HIV-1 infection: a multicentre, double-blind randomised controlled trial. Lancet 2009; 374: 796-806. doi:10.1016/ S0140-6736(09)60918-1

15. Health Outcomes International Pty Ltd in association with National Centre in HIV Epidemiology and Clinical Research. The impact of HIV-AIDS in NSW: mortality, morbidity and economic impact. Sydney: NSW Health; 2007. Available at: http://www.health.nsw.gov.au/resources/ publichealth/sexualhealth/ImpactStatement_pdf.asp (Cited 10 March 2010.)

16. Wilson DP, Hoare A, Regan DG, Law MG. Importance of promoting HIV testing for preventing secondary transmissions: modelling the Australian HIV epidemic among men who have sex with men. Sex Health 2009; 6(1): 19-33. doi:10.1071/ SH08081 